\title{
Not everybody is going to be happy when the catheter comes out early: Can we predict who these people are?
}

\author{
Chi-Fu Jeffrey Yang, MD
}

From the Department of Surgery, Duke University Medical Center, Durham, NC.

Disclosures: Author has nothing to disclose with regard to commercial support.

Received for publication March 19, 2018; accepted for publication March 22, 2018; available ahead of print April $24,2018$.

Address for reprints: Chi-Fu Jeffrey Yang, MD, Duke University Medical Center, Box 3443, Durham, NC 27710 (E-mail: chifu.jeffrey.yang@dm.duke.edu).

J Thorac Cardiovasc Surg 2018;156:436-7

$0022-5223 / \$ 36.00$

Copyright (c) 2018 by The American Association for Thoracic Surgery

https://doi.org/10.1016/j.jtcvs.2018.03.081

The Surgical Care Improvement Project Inf-9 guidelines, established by the US Centers for Medicare and Medicaid Services in 2009 and The Joint Commission, ${ }^{1}$ recommend the early removal of urinary catheters placed at the time of surgery by postoperative day 2 . These guidelines were based on orthopedic, cardiac, vascular, urologic, and gastrointestinal surgical data, ${ }^{2}$ and were not based on outcomes of patients undergoing major thoracic surgery, many of whom have thoracic epidurals to reduce postoperative respiratory complications and postthoracotomy pain. In patients undergoing major thoracic surgery who received thoracic epidural blockade, there have been 3 randomized trials comparing early versus standard urinary catheter removal. $^{3-5}$ These studies have reported conflicting results. The optimal timing of urinary catheter removal is therefore still being debated.

In their study reported in this issue of the Journal, Young and colleagues ${ }^{6}$ set out not to determine whether early removal is better than standard removal, but rather to determine who is most likely to benefit among patients receiving thoracic epidural blockade who have their urinary catheters removed early, within 48 postoperative hours. It is a commonly asked clinical question with limited thoracic surgery-specific data to help answer it.

Young and colleagues ${ }^{6}$ performed a prospective observational study of 288 consecutive patients who had undergone major pulmonary resection by thoracotomy or open esophageal resection with thoracic epidural analgesia from 2013 through 2015. In accordance with Surgical Care Improvement Project Inf-9 guidelines, all urinary catheters were removed within 48 hours after surgery. Of the 275 patients who met study inclusion criteria, 60 patients $(22 \%)$ had catheters reinserted. Of these patients, a much higher percentage of patients undergoing esophagectomy (45\%) required catheter reinsertion than did patients undergoing pulmonary resection $(15 \%)$.

There were no significant differences observed in urinary tract infection incidence between the reinsertion group $(1 / 60 ; 1.7 \%)$ and the group that did not require reinsertion

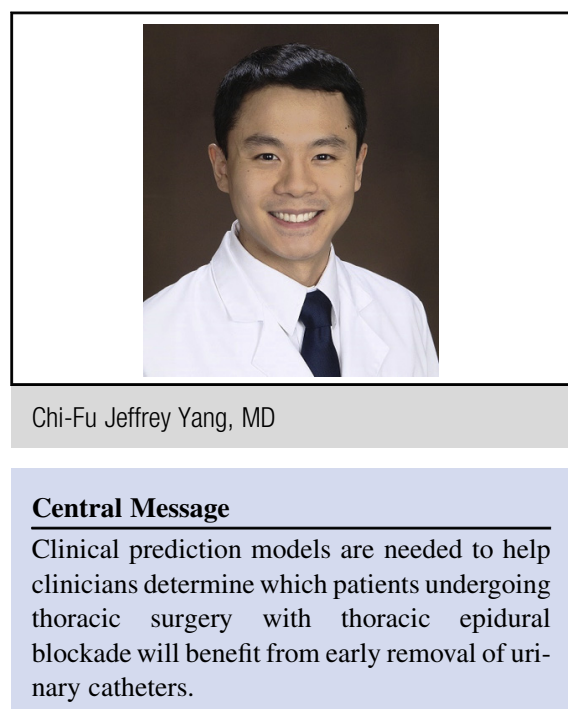

See Article page 430

$(1 / 215 ; 0.5 \%)$. In the catheter reinsertion group, only 1 of $60(1.7 \%)$ patients had urethral trauma from catheter insertion; in this 1 patient, the hematuria resolved without further intervention. In a multivariable logistic regression model, esophagectomy, benign prostatic hyperplasia, and lower body mass index were found to be independent factors significantly associated with urinary catheter reinsertion after early urinary catheter removal.

Young and colleagues ${ }^{6}$ are to be congratulated for performing a well-designed study. The study had numerous strengths. It was prospective, not retrospective, and had well-defined criteria. The protocols are clear and appear to have been followed strictly, with minimal variability. The mixture of opioid and local analgesic in the epidural infusion was consistent, and there was a clear definition of when the urinary catheters were to be removed. All epidural catheters were placed at the same $\mathrm{T}$ level for all procedures. The statistical analysis was also sound and included models that adjusted for confounders and checked for multicollinearity.

The limitations were appropriately acknowledged by Young and colleagues. ${ }^{6}$ First, the sample size was small. Larger numbers might have been able to detect small but significant differences in urinary tract infection or urethral reinsertion trauma. Second, there was a lack of data regarding pain scores and narcotic use. Future studies should include these variables, because the efficacy of the 
epidural analgesia and the amount of opioids received could affect detrusor function. Third, there were no data on previous history of urinary retention, on how benign prostatic hyperplasia was diagnosed, and on whether the patients who had benign prostatic hyperplasia had undergone any previous prostate surgery (eg, transurethral resection of the prostate). These variables, which could be important confounders, are currently missing in the study's model.

In response to the study findings, Young and colleagues ${ }^{6}$ have changed their own institution's practice. Currently, for patients undergoing esophagectomy or with a history of benign prostatic hyperplasia, Young and colleagues ${ }^{6}$ wait to remove urinary catheters until after discontinuing the epidural analgesia. For all other patients, they remove urinary catheters within 48 hours after surgery. This is a reasonable interpretation of the study findings. It is important to recognize, however, that the study does not provide clinicians with specific estimates for individual patients. This particularly becomes an issue for patients who have a combination of low-risk and high-risk factors. Take, for example, a younger female patient, thought to be at low risk for urinary retention after urinary catheter removal, who receives thoracic epidural analgesia and who undergoes an esophagectomy, a procedure that in this study was associated with a higher risk of urinary retention. What is her risk of urinary retention, what is her risk of having a urinary tract infection develop, and should she still have her catheter removed early? The study by Young and colleagues $^{6}$ is not designed to answer these questions. In the future, it will be important to develop and validate accurate clinical prediction models to help physicians determine which patients undergoing major noncardiac thoracic surgery who receive thoracic epidural blockade are most likely to benefit from the early removal of urinary catheters. Equally important will be the continued development of new analgesics, early recovery protocols, and multimodality pain treatment strategies that may avoid epidural analgesia altogether, which may lead to decreases in postoperative urinary retention and other complications. ${ }^{7-11}$

\section{References}

1. Owen RM, Perez SD, Bornstein WA, Sweeney JF. Impact of surgical care improvement project Inf-9 on postoperative urinary tract infections: do exemptions interfere with quality patient care? Arch Surg. 2012;147:946-53.

2. Centers for Medicare \& Medicaid Services/The Joint Commission. SCIP-Inf-9: urinary catheter removed on postoperative day 1 or 2 with day of surgery being day zero. Available at: http://www.hospitalsafetygrade.org/media/file/ SCIPINF9.pdf. Accessed March 11, 2018.

3. Chia YY, Wei RJ, Chang HC, Liu K. Optimal duration of urinary catheterization after thoracotomy in patients under postoperative patient-controlled epidural analgesia. Acta Anaesthesiol Taiwan. 2009;47:173-9.

4. Zaouter C, Kaneva P, Carli F. Less urinary tract infection by earlier removal of bladder catheter in surgical patients receiving thoracic epidural analgesia. Reg Anesth Pain Med. 2009;34:542-8.

5. Allen MS. Video-assisted thoracoscopic surgery versus open lobectomy for lung cancer: time for a randomized trial. Eur J Cardiothorac Surg. 2017; $51: 175$.

6. Young J, Geraci T, Milman S, Maslow A, Jones RN, Ng T. Risk factors for reinsertion of urinary catheter after early removal in thoracic surgical patients. $J$ Thorac Cardiovasc Surg. 2018;156:430-5.

7. Girsberger SA, Schneider MP, Löffel LM, Burkhard FC, Wuethrich PY. Effect of thoracic epidural ropivacaine versus bupivacaine on lower urinary tract function: a randomized clinical trial. Anesthesiology. 2018;128:511-9.

8. Yeung JH, Gates S, Naidu BV, Wilson MJ, Gao Smith F. Paravertebral block versus thoracic epidural for patients undergoing thoracotomy. Cochrane Database Syst Rev. 2016;2:CD009121.

9. Ljungqvist O, Scott M, Fearon KC. Enhanced recovery after surgery: a review. JAMA Surg. 2017;152:292-8.

10. Martin L, Sarosiek B, Harrison M, Hedrick T, Isbell J, Krupnick A, et al. Implementing a thoracic enhanced recovery program: lessons learned in the first year Ann Thorac Surg. March 3, 2018 [Epub ahead of print].

11. Rice DC, Cata JP, Mena GE, Rodriguez-Restrepo A, Correa AM, Mehran RJ Posterior intercostal nerve block with liposomal bupivacaine: an alternative to thoracic epidural analgesia. Ann Thorac Surg. 2015;99:1953-60. 